\title{
Design and Implementation of a Social Network for Laboratory Researchers
}

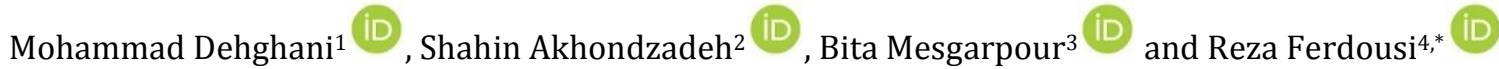 \\ ${ }^{1}$ Scientific and Educational Center for Health Management of Iran, Student Research Committee, Faculty of Management and Medical Informatics, Tabriz \\ University of Medical Sciences, Tabriz, Iran \\ 2 Psychiatric Research Center, Roozbeh Hospital, Tehran University of Medical Sciences, Tehran, Iran \\ ${ }^{3}$ National Institute for Medical Research Development (NIMAD), Tehran, Iran \\ ${ }^{4}$ Department of Health Information Technology, Faculty of Management and Medical Informatics, Tabriz University of Medical Sciences, Tabriz, Iran
}

* Corresponding author: Reza Ferdousi, Department of Health Information Technology, Faculty of Management and Medical Informatics, Tabriz University of Medical Sciences, Tabriz, Iran. Tel: +989144024707; Email: ferdousi.r@gmail.com

Received 2020 August 13; Revised 2020 September 15; Accepted 2020 0ctober 06.

\begin{abstract}
Background: One of the ways to reduce the impact of sanctions on health-related issues is to rely on domestic researchers; however, there are obstacles in the way of researchers in Iran. One of the solutions that can solve their problems is to use a national academic social network. Objectives: This study aimed to describe the procedure of the implementation of an academic social network in a developing country in four stages.

Methods: Given that there has been no previous research in this regard, the present study was the first attempt to design and implement a social network for Iranian health academics. Therefore, to achieve the goals of this study, various methods will be used to prevent the failure of the project and gather comprehensive information. This research project will make use of four qualitative studies, one comparative study, one review study, and four quantitative studies.

Results: Results: This study introduces a model for an academic social network for health researchers. This model consists of four main steps and 13 sub-steps. It should also be noted that the needs assessment and determination of the usability and performance are among the strengths of this model.

Conclusion: This study faces two main challenges, namely the lack of budget and similar national social networks. Moreover, the problems of information and communication technology infrastructure in Iran and issues, such as compliance with copyright and privacy, complicated the project.

Keywords: Academic social network, Health research, Research network, University social network
\end{abstract}

\section{Background}

In November 2018, the U.S. government abandoned the international agreement registered at the United Nations and imposed more severe sanctions on Iran (1). This agreement about the nuclear protocol was signed by Iran and the P $5+1$ on July 20, 2015, according to which the sanctions imposed on Iran in 2012 due to nuclear activities should have been canceled (2).

During the reduction of gross domestic product (GDP) in 2012-15 caused by sanctions, the health system in Iran faced several challenges due to its strong dependence on government spending (3). The sanctions imposed in 2018 were even more severe than those imposed in 2012; therefore they may cause even more challenges $(1,4)$. The most critical problems of this period included the provision of essential medicines, medicines for special diseases, and laboratory and hospital equipment $(5,6)$. Inability of pharmaceutical companies to trade with international companies and the problems of money transfer as well as the fear of international companies of the penalties of the U.S. government have aggravated the crisis (6-8).
Imposition of sanctions is a cheaper form of war with less human costs which is also more politically acceptable (9). The countries that are subject to sanctions should reduce the harmful effect of sanctions by using their domestic resources. Iran also has attempted to provide healthcare resources relying on its infrastructures. Iran has 52 medical universities (ranked $16^{\text {th }}$ in the world regarding its population) (10), 180 research institutions, and 729 health research centers (11). In addition to its health infrastructures, Iran is one of the top countries in the Middle East regarding specialized human resources. In 2015, 4.5 million Iranian students were studying at academic institutions. In 2012 (the first year of sanctions), 54800 researchers $(0.7 \%$ of the world's researchers) lived in Iran. In 2016, Iran ranked $16^{\text {th }}$ globally in science production, with 51158 papers published in Scopus indexed journals (12).

Reliance on Iranian scholars and scientists is the best strategy to confront the effects of sanctions on the health system; however, Iranian scholars faced substantial challenges in this path. Due to the first group of challenges caused by sanctions, even reputable journals rejected the papers authored by Iranian scholars $(8,13)$. Moreover, due to the 
devaluation of the Rial, Iranian scientists and researchers are not able to supply laboratory resources and equipment anymore and face difficulties for participation in international scientific conferences (14). Second group of challenges is related to the interaction among Iranian scholars, which includes less teamwork, lack of access to shared resources, and lack of utilization of their skills (15). Third group of challenges facing Iranian scholars is concerned with the provision of the necessary research resources which can be due to several reasons, such as the poor distribution of laboratory facilities, national currency devaluation, rise in inflation, reduced research per capita due to GDP reduction, and bureaucracy-related problems (16).

Today, academic social networks are specialized to support researchers in various fields (17). For example, Mendeley makes it possible to search and share papers (18), Academia.edu is designed to facilitate interaction among scholars (19), and ResearchGate allows the exchange of knowledge and skills between the researchers $(17,18)$. Educational role of social networks in academic environments includes the exchange of general information among users, awareness raising, provision of specialized and educational training, provision of professional educational advice, announcement of the programs of virtual and in-person educational institutions, provision of support for virtual and in-person educational institutions, establishment of a connection between users and institutional and educational agents, promotion of virtual education, and provision of specialized training (20). Furthermore, social networks are used in academic communities to facilitate communication with domestic and international professors, identify researchers, participate in research, share scientific resources, exchange document or data resource, ask questions and requests, publish scientific studies, find scientific resources, and provide/obtain data (21).

As noted above, given the current situation of Iran, scholars face several challenges, such as the distribution of research resources and materials. In addition, the sanctions have made it difficult to import research materials and equipment. The problems caused by the rise of inflation and national currency devaluation have led the research directors and researchers to reform distribution networks of research resources, optimize resource usage, and share resources.

Utilization of an academic social network is the best solution for this issue. Academic social networks can be used by health researchers to overcome these problems, accelerate the research activities of Iranian scholars, and reduce the effects of sanctions on the health system of the country. Currently, there are no academic social networks facilitating resource sharing for scholars.
Given the situation of Iran, using a national academic social network can be highly effective in solving the problems of health scholars. In this regard, a Ph.D. thesis entitled "Design and Implementation of a Social Network for Health Researchers" (ethics code: I.R.TBZMED.REC.1398. 184, available on http://ethics.research.ac.ir/ IR.TBZMED.REC.1398.184) aimed to design a national academic social network for Iranian health scholars.

\section{Objectives}

This study aimed to describe the procedure of the implementation of an academic social network in a developing country in four stages.

\section{Methods}

As mentioned above, the current project is the first attempt to design a social network for Iranian health scholars, and there is no previous study in this regard. Therefore, to achieve the goals of this study, the authors will use various methods to prevent the failure of the project and collect comprehensive information.

In the first step, to identify the needs of this academic social network, the authors will interview health researchers and supplement the collected data by reviewing related articles. They will also compare academic social networks to enrich the data and finally, after the omission of the duplicate data, the findings of this step will be presented to a group of experts for prioritization.

The second step was designed based on the limited budget of this project and the different infrastructures of information and communication technology (ICT) in Iran. In this step, the Delphi technique will be used to determine the appropriate infrastructure for the creation of this social network. In the third and fourth steps, the designed academic social network will be evaluated using different methods (Figure 1).

\subsection{Step 1: Needs assessment for the academic social network}

A combination of the following three methods will be applied for the comprehensive data needs assessment and determination of the performance of this academic social network.

A) Needs assessment based on interviews

In this step, the team will prepare interview questions (based on guidelines) based on the research objectives. Needs of health researchers and research managers with at least two years of experience who have used academic social networks will be extracted using a qualitative method (i.e., semi-structured interview). The inclusion criteria are a history of health research activities and utilization 


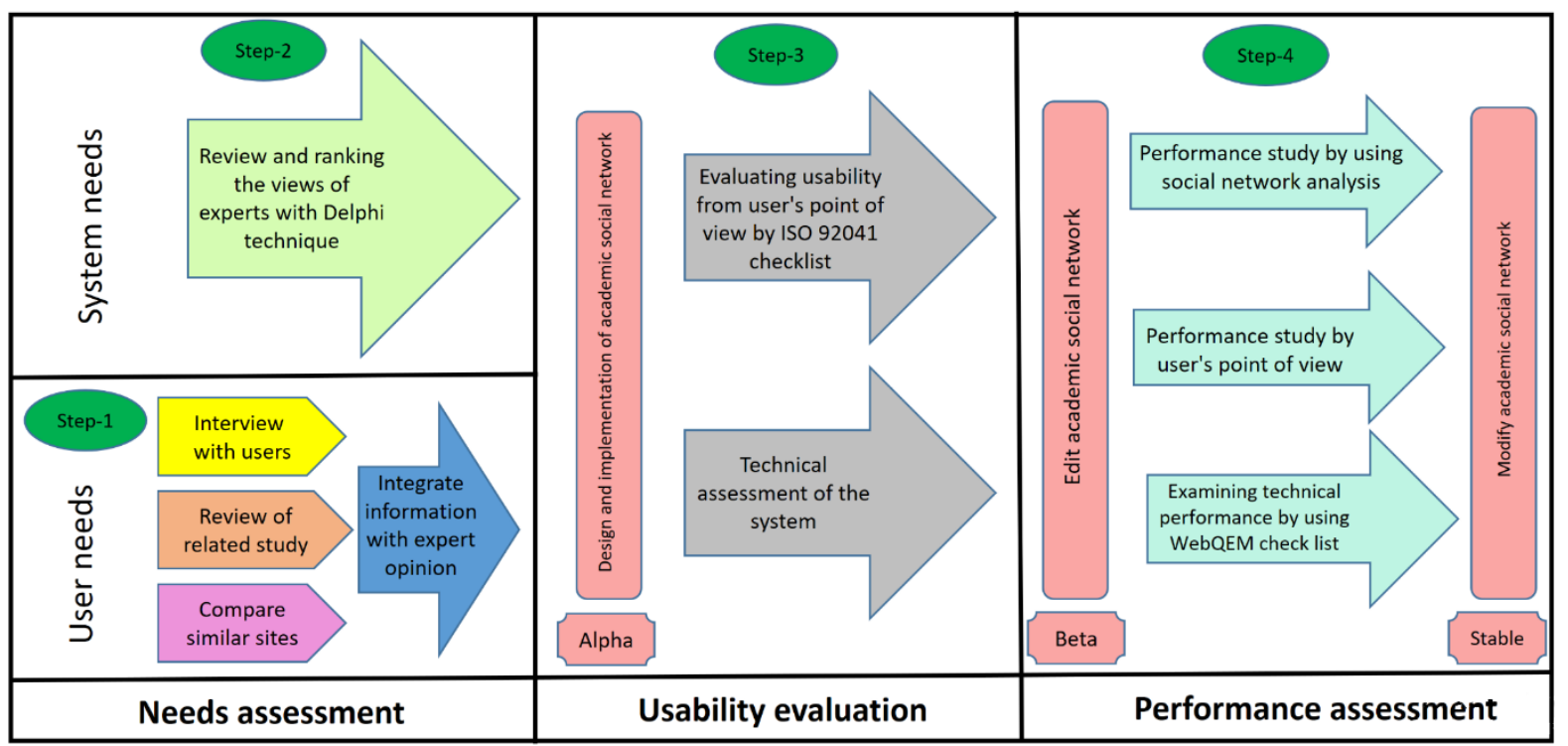

Figure 1. Model of Design and Implementation of a Social Network for Laboratory Researchers

of academic social networks. The samples will be selected using the purposive sampling method.

The interviews will be recorded, with the permission of participants, and subsequently transcribed. The Corbin-Strauss constant comparative method will be used for data analysis. This analysis method includes three steps of open, axial, and selective coding.

B) Needs assessment using a comprehensive review

In this step, the authors will review the papers about academic social networks. A search strategy will be constructed using suitable keywords, and then Embases, Medline, and Cochrane databases will be searched. Besides, for further searching, Pubmed, Web of Science, Google Scholar, and Scopus will be used, and the resources of the extracted papers will be reviewed as well. After the determination of the related literature, their data will be extracted, classified, and reported in tables.

C) Needs assessment using a descriptive comparative study

In this step, the authors will investigate and compare the structure, performance, and user interface of the five most popular global academic social networks. In addition to websites, the latest editions of manuals and documents provided by the creators of these academic social networks will be investigated to extract data. The obtained data will be entered into a table based on research objectives which subsequently will be analyzed using descriptive statistics. Finally, the results will be summarized in tables.

D) Prioritization of the system needs using an expert panel

In this step, the data obtained from the three previous steps will be integrated and the duplicate data will be deleted. Afterward, the remaining data will be prioritized using the panel of experts. In total, 15 experts in the fields of research management and design of information systems and social networks will be selected using a purposive sampling method. Moreover, it is noteworthy that the sessions will be held based on scientific methods.

At the end of each session, the experts will be asked to fill out a checklist to evaluate the importance and feasibility of the system needs based on a scale ranging from very important to unnecessary. The score of each item will be calculated by multiplying the score of importance by the score of feasibility and the items with a mean score of less than five will be excluded from the study.

\subsection{Step 2: Determination of the infrastructure} required for the implementation of an academic social network

The imposed sanctions and the particular situation of ICT infrastructure of Iran led the authors of this research to determine the suitable infrastructures for academic social networks using library studies and expert interviews. Furthermore, the selected infrastructures were prioritized using the Delphi technique. In total, 25 participants will use the Delphi technique, and the specified infrastructures will be ranked in three steps. Finally, the infrastructure required for the academic social network will be determined based on the condition of ICT infrastructure in Iran.

\subsection{Step 3: Evaluation of the usability of the academic social network}

In this step, the academic social network will be designed and coded according to the needs and performance specified in the first step and the 
determined infrastructure in the second step. Subsequently, the system will be evaluated based on its usability from the user's point of view and its compliance with technical standards; thereafter, potential system issues will be fixed.

A) Evaluation of the usability from the user's point of view

In this step, system usability will be evaluated from the point of view of users using section 10 of the ISO 92041 checklist. This tool includes 50 questions about usefulness, ease of use, ease of learning, satisfaction, display, system terminology and information, user personalization, error management, and system capabilities.

B) Technical assessment of the system

In this step, the technical specifications of the website will be evaluated using appropriate tools. To this end, the URL or the source code of the social network will be given to analysis programs or websites for security considerations; consequently, the problems will be detected and solved.

\subsection{Step 4: Examination of the performance of the academic social network}

After solving system problems in step 3, the social network will be implemented on a dedicated server according to the priorities set out in steps 1 and 2 . Moreover, the marketing will be done differently and after three months, the performance of this social network will be examined.

A) Examination of the social network performance using social network analysis

In this step, social network analysis will be used to collect and analyze data and predict social structures and discover communication patterns among users. The required data will be extracted from the social academic network database and will be entered into GeForce, NetDraw, and Pajek programs after preparation.

B) Examination of the social network performance from the point of view of users

In this step, the active users of this social network will be identified and their views on the performance of the social network will be examined using interviews. The interview will last $30 \mathrm{~min}$ and the number of participants will depend on the collected data. In each interview, demographic questions will be asked, the research objective will be described to the participants, and the interview will be recorded with their permission. Thereafter, the interviews will be typed and encoded. As it is common in qualitative analysis, the data collection and analysis will be performed simultaneously. The Corbin-Strauss constant comparative method will be used for data analysis. This analysis method includes three steps of open, axial, and selective coding.

C) Examination of the technical performance of the social network

In this step, the performance of the designed academic social network will be compared to other domestic and international academic social networks. WebQEM will be used for this comparison, which evaluates the usability, functionality, reliability, and performance of the website according to the ISO 9126 checklist from IEEE Std 1061. The data collected from step 4 will be used to improve the performance of the system.

In accordance with informed consent procedures, the research participants will be provided with a brief verbal explanation of the study and told that they can leave the study at any time. As mentioned, the ethics committee of Tabriz University of Medical Sciences has approved this study in (code: IR.TBZEDMED.REC.1398.184).

\section{Results}

Today, information and communication technology has caused many changes in human life. One of these technologies is the Internet, which has led to the creation of various social media. A social network is a type of social media that is used by researchers as well as the rest of society.

Specialized social networks for researchers are called academic social networks and help the researchers connect with each other and enhance collaboration between them. In addition, researchers use this social media to share research resources and promote their research activities. This web-based tool helps researchers identify their peers and ask questions from them if needed. Each academic social network is created for a specific purpose and has its own users. They might have been created to share research resources, enhance collaboration, manage print resources, and provide intelligent information.

There is no national academic social network in Iran and this can reduce the efficiency of universities and researchers. Activities of health researchers are of particular importance since they can have a significant impact on community health.

In recent years, economic problems and sanctions have caused various problems for health researchers in Iran. Accordingly, the design and implementation of this academic social network can resolve some of the problems of health researchers in this country. This social network introduces Iranian researchers to each other and is also a suitable platform for questions and answers, material sharing, and enhancement of the efficiency of research equipment.

This academic social network was designed and implemented in four main steps. In the first step, the needs of health researchers will be identified using various methods, such as interviews with them. In the second step, according to the specific conditions of Iran in terms of ICT, the required infrastructure for a social network will be determined. In the 
second and third steps, the usability and performance of the system will be evaluated to increase user satisfaction.

\section{Discussion}

This academic social network is expected to improve interaction among Iranian health scientists and scholars and also reduce the issues related to the distribution of laboratory materials and equipment. Besides, senior health managers can use research tools, such as social network analysis and data mining to detect problems more quickly. The authors predict that some limitations will be encountered in the design and implementation of this academic social network. For example, considering the grant given to the project (450 U.S.\$), the researchers may face funding problems in different steps. Moreover, they may face significant challenges caused by sanctions regarding the provision of infrastructures, such as the required software and hardware. Another potential challenge for this social network is the lack of trust in a domestic academic social network.

\section{Conclusion}

The design and implementation of this specialized social network for health researchers encompasses a complete process ranging from needs assessment to usability and technical performance. The needs assessment performed in the first step reduces the risk of system failure and examination of the appropriate infrastructure in the second step can prevent future problems, such as lack of bandwidth or storage space.

This design and implementation model, in addition to health portals and websites, can be a good model for designing treatment and health software and application.

\section{Acknowledgements}

The authors would like to appreciate the Research and Technology Deputy at Tabriz University of Medical Sciences for supporting this project.

\section{Footnotes}

Authors' Contribution: All authors were involved in the design of the study and will analyze each piece of data in the future according to their expertise. All authors have read and approved this manuscript.

Conflict of Interests: The authors state that there was no conflict of interest in this article.

Ethical Approval: In accordance with informed consent procedures, the research participants will be provided with a brief verbal explanation of the study and their written consent will be obtained. Moreover, before the interview, they will be told that they can leave the study at any time. As mentioned, this study has been approved by the ethics committee of Tabriz University of Medical Sciences (code: IR.TBZEDMED.REC.1398.184).

Funding/Support: This research is derived from a dissertation submitted in partial fulfillment of the requirement for the degree of Ph.D. that was financially supported by the Research and Technology Deputy of Tabriz University of Medical Sciences. It should be mentioned that this study did not receive external funding.

\section{References}

1. Aloosh M, Salavati A, Aloosh A. Economic sanctions threaten population health: the case of Iran. Public Health. 2019; 169:10-3. doi: 10.1016/j.puhe.2019.01.006. [PubMed: 30771721].

2. Mucci N, Giorgi G, Roncaioli M, Perez JF, Arcangeli G. The correlation between stress and economic crisis: a systematic review. Neuropsychiatr Dis Treat. 2016;12:983-93. doi: 10.2147/NDT.S98525. [PubMed: 27143898].

3. Gorji A. Sanctions against Iran: the impact on health services. Iran J Public Health. 2014;43(3):381-2. [PubMed: 25988101].

4. Aloosh M. How economic sanctions compromise cancer care in Iran. Lancet Oncol. 2018;19(7):e334. doi: 10.1016/S14702045(18)30427-3. [PubMed: 30084377].

5. Hosseini SA. Impact of sanctions on procurement of medicine and medical devices in Iran; a technical response. Arch Iran Med. 2013;16(12)736-8. [PubMed: 24329148].

6. Massoumi RL, Koduri S. Adverse effects of political sanctions on the health care system in Iran. J Glob Health. 2015; 5(2):020302. doi: 10.7189/jogh.05-020302. [PubMed: 26207178].

7. Karimi M, Haghpanah S. The effects of economic sanctions on disease specific clinical outcomes of patients with thalassemia and hemophilia in Iran. Health Policy. 2015;119(2):239-43. doi: 10.1016/j.healthpol.2014.12.011. [PubMed: 25564279].

8. Shariatirad S, Maarefvand M. Sanctions against Iran and the impact on drug use and addiction treatment. Int J Drug Policy. 2013;24(6):636-7. doi: 10.1016/j.drugpo.2013.04.003. [PubMed: 23683411].

9. Arya N. Economic sanctions: the kinder, gentler alternative? Med Confl Surviv. 2008;24(1):25-41. doi: 10.1080/136236 90701775205. [PubMed: 18456989].

10. Tavakol M. Medical education under economic sanctions in Iran. Acad Med. 2009;84(10):1324. doi: 10.1097/ACM.0b013e 3181b6552b. [PubMed: 19881411].

11. Akhondzadeh S, Ebadifar A, Baradaran Eftekhari M, Falahat K. Medical science and research in Iran. Arch Iran Med. 2017;20(11):665-72. [PubMed: 29480730].

12. Farhadi M. Iran, science, and collaboration. Science. 2015;349(6252):1029. doi: 10.1126/science.aad3016.

13. Saeidnia S, Abdollahi M. Consequences of international sanctions on Iranian scientists and the basis of science. Hepat Mon. 2013;13(9):e14843. doi: 10.5812/hepatmon.14843. [PubMed: 24282428].

14. Arie S. Unintended consequences of sanctions against Iran. BMJ. 2013;347:f4650. doi: 10.1136/bmj.f4650. [PubMed: 23881946].

15. Cheraghali AM. Scientific apartheid. Hepat Mon. 2012; 12(3):158-9. doi: 10.5812/hepatmon.841.

16. Soofi A, Ghazinoory S. Science and innovations in Iran: development, progress, and challenges. Berlin, Germany: Springer; 2013.

17. Jeng W, DesAutels S, He D, Li L. Information exchange on an academic social networking site: a multidiscipline comparison on researchgate Q\&A. J Assoc Inf Sci Technol. 2017;68(3):63852. doi: 10.1002/asi.23692.

18. Jeng W, He D, Jiang J. User participation in an academic social 
networking service: a survey of open group users on Mendeley. J Assoc Inf Sci Technol. 2015;66(5):890-904. doi: 10.1002/ asi.23225.

19. Hammook Z, Mišić J, Mišić VB. Student/supervisor collaboration and usage patterns of publications available on ResearchGate. 2016 IEEE Wireless Communications and Networking Conference, Doha, Qatar; 2016. P. 1-5. doi: 10.1109/WCNC.2016.7564814.
20. Ahn J. The effect of social network sites on adolescents' social and academic development: current theories and controversies. J Am Soc Inf Sci Technol. 2011;62(8):1435-45. doi: 10.1002/asi.21540.

21. Williams AE, Woodacre MA. The possibilities and perils of academic social networking sites. Online Inform Review. 2016;40(2):282-94. doi: 10.1108/OIR-10-2015-0327. 